\title{
Unpacking | A Study in the Generation of Louis Sullivan's Ornament
}

\author{
NICHOLAS AULT \\ Sodium Design
}

The ability for students to understand drawing as an analytical method and apply it in an exploratory and/or analytical manner is critical in their fundamental development and their understanding of Architecture. Difficulty arises because a clear implementation and articulation of this abstract idea as a technique is difficult, especially when dealing with a large group of students. The development of a curriculum that assists the students in understanding the investigative nature of drawings and the discipline of articulating drawings relies heavily on a systematic approach that values graphic relationships and rigorous logic. By utilizing Louis Sullivan's quasi-algorithmic approach to generating ornament as the precedent and methodology the students are able to logically take apart a these constructs and understand the power of drawing both as an investigative tool, representational device and generative language.

Utilizing precedent is fundamental to the understanding of architecture and a foundational element in architectural education. Sadly, from an academic standpoint, this endeavor generally lacks rigor and seems generally disconnected from the creative aspects of architecture as anticipated by students. As a practice, the study of precedent allows for complex schema to be pointedly distilled so that a critical understanding of an architectural construct - canonical, prototypical, categorical, or representative - can be digested and understood on a level not visually apparent in its fully rendered form. The work of Louis Sullivan, specifically the procedure developed to create exterior ornamentation, is uniquely suited to precedent study and analysis. Generated utilizing a quasi-algorithmic method, Sullivan's ornamentation can be capably digested utilizing contemporary techniques and processes intrinsic to computer software that assist in bridging the historic divide between precedent and student understanding. By implementing this type of study as part a foundation course the students are able to learn contemporary skills in drawing, modeling and theory by means of precedent analysis from a project and methodology employed a century ago.

The profession of architecture, while constantly engaged in an ever-evolving state of flux, still fundamentally communicates through drawing. The formal nature of this communication methodology is shifting but the ability to abstract complex information fundamentally still forms a critical element of built architecture. Meanwhile, the ability for students to demonstrate a mastery of communication is key and the ability to see, quickly understand and express complex information in a facile manner is critical. As the architectural educational system has mostly accepted digital communication a primary language, the manner in which architectural thought is communicated to the students has become a sticking point in many programs - given that the students predilection for three-dimensional visualization has outpaced the development of pedagogical techniques at many universities. Yet, the ability for students to understand drawing as an investigative tool and employ it as means for discovery is critical to their growth and understanding of precedent.

The development of projects that assists the students in understanding the abstract and investigative nature of drawings relies heavily on a process that values graphic relationships, proportion, formal associations, rigor and embedded logic - not dimensional measurements and scale. Through the investigation and analysis of the ornamental designs of Louis Sullivan a concise and ordered process of investigation can be achieved by the application and guidance of Sullivan's 'seed germ' concept. These compositions, devoid of a measurable scale carefully reconstruct an underlying logic that reinforces the students utilization of the architectural tools of precedent, diagramming, lineweight, logic, process and investigation. This 'unpacking' implements an investigative and rigorous approach that elicits a complex aggregation of simplistic geometric interrelationships that reconstitute the complexity clearly evident in Sullivan's ornamental work.

\section{SULLIVAN AND THE SEED GERM}

Louis Sullivan's buildings have been widely studied and are renowned for their clarity and logic as well as a visual elegance that demonstrates a set of parameterized characteristics that evolved from an understanding of abstracted historical precedent. Sullivan's ornament, on the other hand, is a unique derivative of geometry and a procedure seemingly unique to his process, tools and ideology. Sullivan has stated that, 'the building's identity resides in the ornament.' ${ }^{1}$ This belief that the understanding of each buildings' ornament leads to a greater understanding of Sullivan's architecture gives a glimpse into his design process. In his 1926 ornament and design treatise, A System of Architectural Ornament According with a Philosophy of Man's Powers, Louis Sullivan outlines a theoretical agenda that gives an insight into the development of his ornamental style and an approach to the holistic generation of an architectural grammar. What becomes apparent when studying this manifesto is how the algorithmic nature 


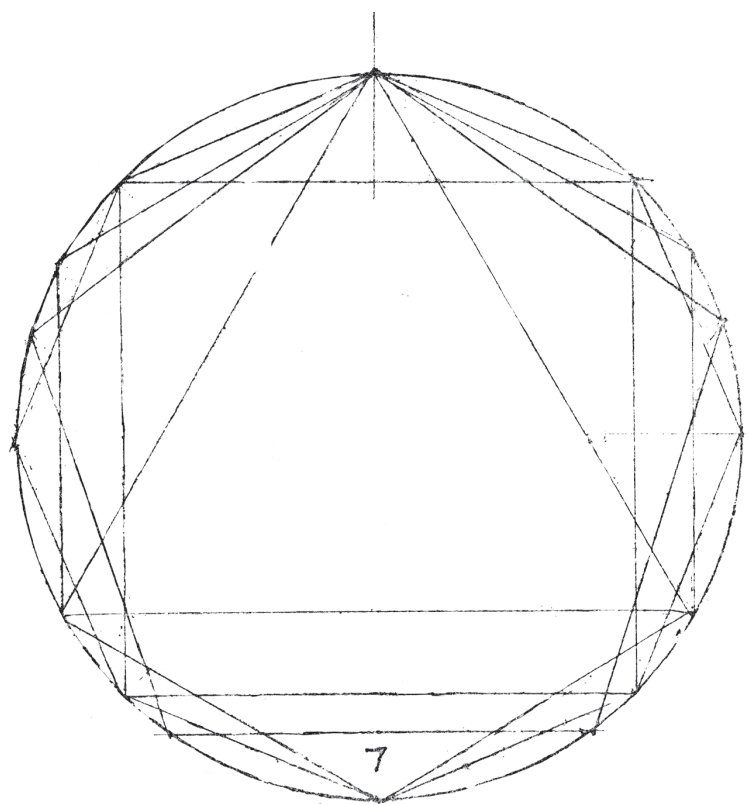

Figure 1. Geometric Inscription - Louis Sullivan. ${ }^{2}$
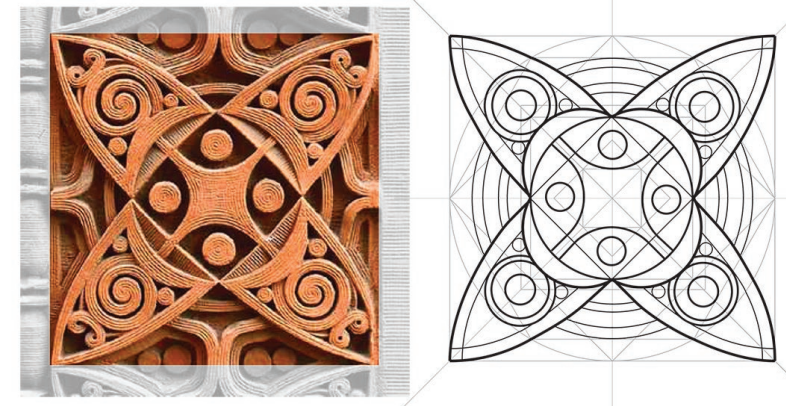

Figure 2. Detail + Analytical Drawing - Entry Portico, Wainwright Building Student: Austin Ferguson.

of each detail is organized and constructed. This geometric logic leverages a simple rule-set of geometric inscription and circumscription, linear proportional divisions, symmetry and radial arrays to create elaborate and elegant compositions.

This linear method of construction makes it possible for one to reconstruct the geometric logic and devolve each design back to its 'seed germ.' This process of de-evolution forms the fundamental analytical goal of this project and underlines how, through diligent investigation, students can analyze a composition (architectural construct) down to its root and understand the fundamental ordering principles and relationships that underlie the final composition.

Constructed as a method to introduce students to the concept of diagramming, precedent analysis, and a variety of software packages this project was designed to turn the tables on the students - they are to become researchers instead of merely creators. Devoid of dimensional information, Sullivan's ornamental compositions are holistically constructed through the interplay of geometry and proportional relationships within the whole.

Louis Sullivan theorizes that these ideals are a response to the dichotomic relationship between the 'organic' and the 'inorganic.' These definitions do not necessarily align with the terminology that we would normally associate with them but Sullivan goes as far to state that:

By the word inorganic is commonly understood that which is lifeless, or appears to be so; as stone, the metals and seasoned wood, clay or the like. But nothing is really inorganic to the creative will of man. His spiritual power masters the inorganic and causes it to live in forms which his imagination brings forth from the lifeless, the amorphous. He thus transmutes into the image of his passion that which of itself has no such power. Thus man in his power brings forth that which hitherto was non-existent.

For man is power, and this power is native in nature with the power of the germ of the seed. Thus he commands at will the realm of the organic or living, and therein again he creates as he wills; for he has the power to will - it is one of his many powers. ${ }^{3}$

In this statement Sullivan clearly aligns man's physical, intellectual, emotional, moral and spiritual self with nature. Man is the manifest nature of the germinated seed - two lobes: one of intellect and the other desire. ${ }^{4}$ This polarized relationship is represented in the complex interplay of hard lined geometry, the inorganic, intertwined and occluded by hypernatural flora, the organic. These two entangled elements, the inorganic and the organic, create a rich visual complexity but the relationship they create exists outside the purview of this assignment and as such, from this point forward the focus of will be the conceptualization, generation and refinement of the inorganic.

Upon removing the flora from the object, a geometric composition is presented and the task becomes finding the geometric and logical 'seed' from which the geometry germinates and expands. This system, uniquely composed in each instance, generates a self-contained genealogy in which the composition of the ornament derivatively devolves into children, grandchildren, great grandchildren and so forth. Already an abstract composition the students must look past the realized three-dimensional information presented and instead, through investigation and construction line relationships, deconstruction the composition until the root elements begin to take shape and the algorithmic base is exposed. This process is not dissimilar to the original undertakings present when first studying the geometric relationships within an 
architectural composition. But, instead of being clouded by preconceived notions held in an understanding of architecture, these constructs can be purely investigated to discover relationships, logic and composition separate from the noise present in the architectural drawings traditionally employed in these early exercises. This geometric investigation empowers the students to become researchers and utilize drawing as a tool from which to uncover a more robust understanding of an object and consequently the larger world around them. This understanding begins the formation of a larger understanding of more contemporary en vogue design principles already in place in the early 21 st century - that of the algorithm and parametric system. The utilization of ornament for Sullivan becomes the algorithmic or fractal-like resolution to a project. He refers to this relationship as organic, in which a buildings overall composition, massing, and detail spiral down into itself not through replication but instead through an almost familial translation of geometric association. The resultant translation begets a family of details that spawn from the same genesis and share characteristics with one another all the while become wholly unique and self-derivative.
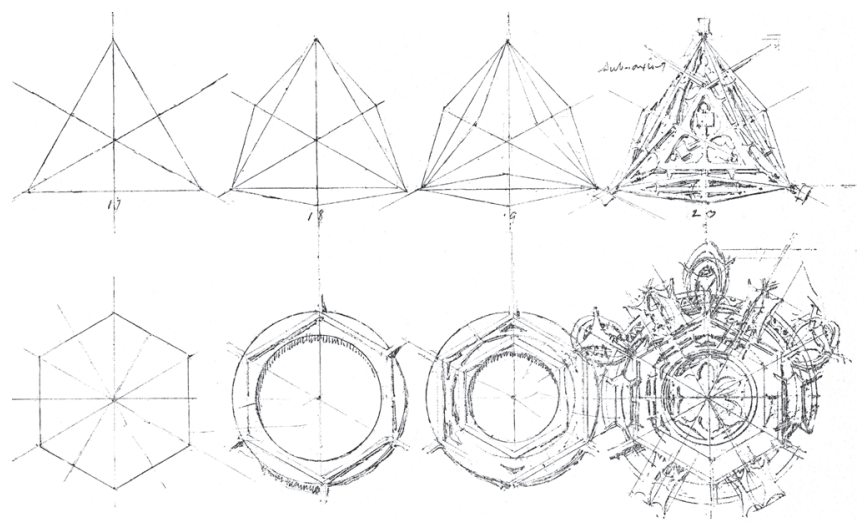

Figure 3. Sample ornament generation showing radial divisions and geometric layering. ${ }^{5}$

\section{SUCCINCTNESS}

The efficiency by which information is created sometimes is not always of critical importance, especially in the digital world. However, it is hard to imagine a world in which concision in the quantity of information that is communicated is not of critical importance. Architecturally we utilize the symbology and semantics of drawing to represent the world but there are few axioms that govern the manner in which this information is derived. In mathematics and the physical sciences the utilization of the principle of Occam's Razor is often employed as a means to simplify calculations or procedures down to their critical elements and refine the purity of an idea, equation or process down to its irreducible threshold. To bring this concept into the arts, the application of Occam's Razor can be simplified a bit further by the statement 'the simplest solution is the most beautiful' or to colloquially quote Mies van der Rohe, 'Less is More.'
The concept of Occam's Razor in this project is of paramount importance. Sullivan's details, layered with filigree and densely packed with geometry, are primed for overwrought analysis, especially by students investigating a built up complexity for the first time. In many ways the beauty of Louis Sullivan's ornament generation comes from a perceived lack of organization or process when in fact there is a highly structured and principled underlying structure. Assuming that the algorithmic (process) is a method of generation, producing complex forms and structures based on simple component rules. ${ }^{6}$ The algorithmic rules as graphically outlined in his 1924 treatise, A System of Architectural Ornament, explain the framework from which all of his ornamental generation and variation had been derived. As with all algorithms, the configuration of geometrical relationships fundamentally alters the product and in itself becomes open for interpretation. As a key constituent of these investigations, the students drill into this algorithm and regenerate Sullivan's process in as succinct a manner as possible always searching for clarity in their logic and representation with the final incarnation being an accurate re-presentation of the original ornamental construct.

\section{PROCEDURE}

It is said that architects learn to see by drawing. Clearly the re-presentation of the existing is not in itself sufficient to understand and link the built world into our own creative works. The process of drawing itself is a process of interpretation, abstraction and representation with diagramming becoming a formal distillation designed to understand a desired criteria or characteristic. This makes a case for the importance of drawing as analysis. The purpose of analysis is to understand the underlying constituent and workings, so that their powers may be assimilated and acquired. ${ }^{7}$ Analysis is most useful when it provides an understanding of what is possible and develops a framework of ideas for the imagination to work with. ${ }^{8}$

This assignment takes place as a component within a second year undergraduate design studio. Therefore, the specific learning outcomes of this assignment are split. As a computer skills assignment the project is responsible for instructing a variety of tools, in this case Adobe Photoshop, Adobe Illustrator and to some extent Rhino. As a portion of a design studio course, this project serves as an introduction to precedent, drawing generation, abstraction and diagramming. The utilization of the computer in this process is meant to leverage the computer's innate ability to accurately articulate geometry and geometric relationships, representational characteristics and specifically its ability to articulate complex geometries quickly and concisely. A key constituent in all of this is the clear utilization of whole geometries versus the piecemeal approach to polygons generally utilized when conducting investigations by hand. The computer empowers us to utilize mathematically precise polygons of indeterminate measurement while hand drawing requires forethought for composition of individual 
lines. Digital tools capably draw circles, pentagons, hexagons etc. and employ embedded 'snap' capabilities to create precise relationships otherwise difficult to achieve. These object snaps, enable things such as polygonal or circular centers and geometric tangencies to be utilized repeatedly by complimentary geometries. This tool is especially beneficial given the repetition of inscribed and circumscribed geometry commonly utilized in the generation of Louis Sullivan's work.

The utilization of Sullivan's algorithmic approach coupled with the digital tools presents the students with a "kit-ofparts" from which to begin the reconstruction. Consisting of simple geometries - circles, ellipses, quadrilaterals, and an assortment $\mathrm{n}$-gons - the students search for geometric relationships that occur within the detail; typically achieved graphically through the use of a photographic underlay. Gradually geometric aggregation works inward and through repeated inscription from a central point the articulation of the larger geometric composition begins to take shape. As the geometric ornament evolves, smaller relationships become articulated by rectangular and polar arrays and fill out the detail. Similar to the Gothic utilization of daisy wheels, ${ }^{9}$ these smaller constituent parts create angular sections in the geometric layering and define zones (bounding boxes) in which additional geometry populates the outer and intermediate areas of the detail itself.

\section{PRODUCT}

The project itself consisted of three disparate parts. Each part is meant to be an outcome wholly independent from and representing something different about the ornament - each displaying the student's process and the procedure from which they unpacked the ornament itself. These representations featured the implementation and subsequent regression of geometry from object line to construction line. Those purely constructed as geometries acting upon others or as investigative ventures in themselves with no real bearing on the final composition.

The second portion of the assignment focuses expressly on the annunciation of the individual steps themselves. By exploring the individual component relationships through text the students are forced to express their understanding of the composition. This text based articulation forces the students to understand and articulate proportional relationships and geometric generation patterns as the fundamental principle as commands such as, 'offset circle $1 / 4$ "' are deemed unacceptable. By reinforcing the geometric relationships in a verbal manner the students must consider the quantity of information embodied in drawing and communicate it to an audience. This has been conceptualized as a manner for them to explore the generation of the object but also a means for them to evaluate and edit the information being communicated through the drawings.

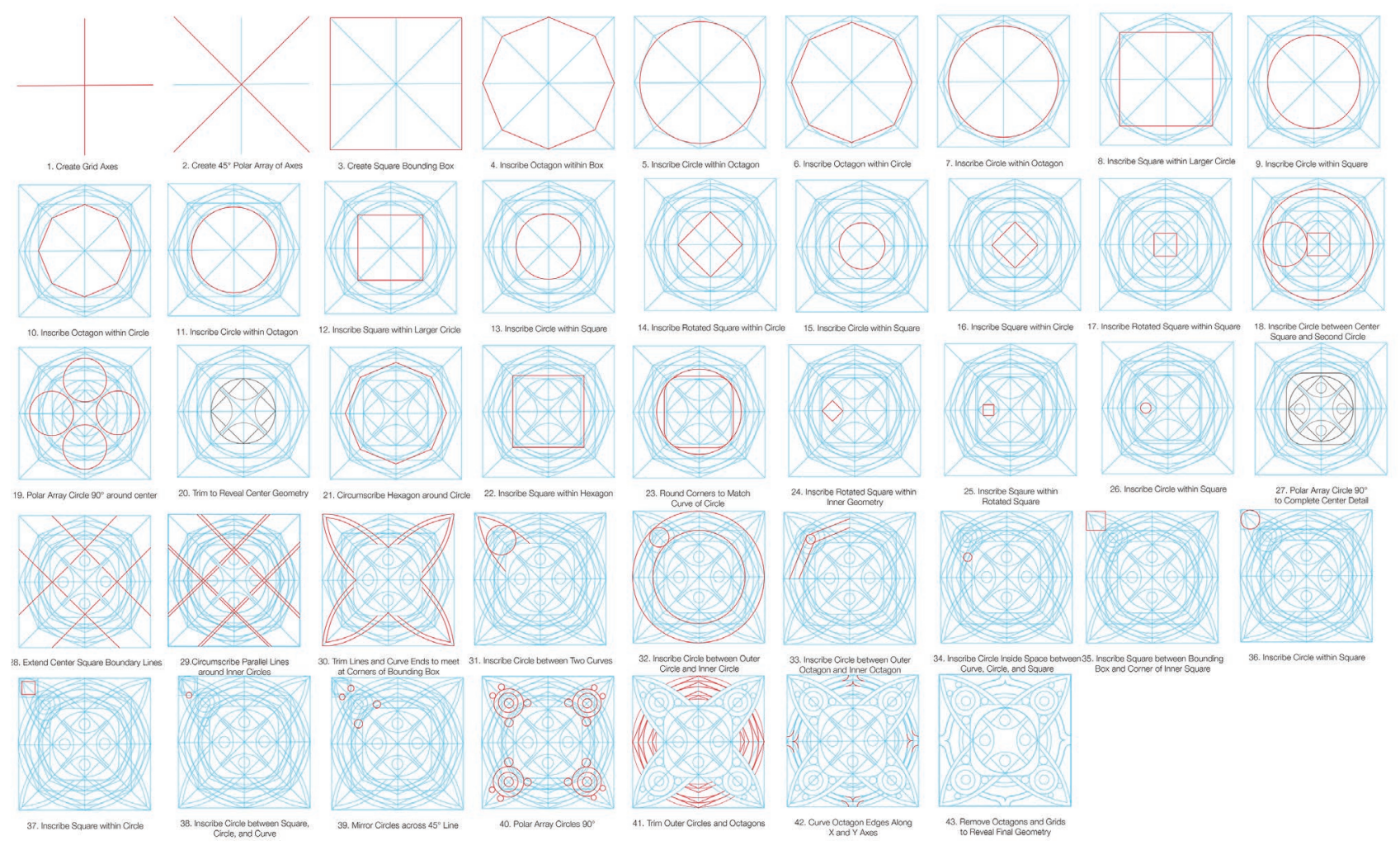

Figure 4: Full analytical Drawing Set - Shawn Backstrom. 
The final requirement for the project is the combination of information through the medium of animation. These simple animations are primarily seen as a technique for presentation but unexpectedly the students utilized the animations as a means to recalibrate their reconstruction process. These animations, simultaneously presenting the graphic and verbal reconstruction process, not only introduce the students to simple tools of presentation but also to animation as a potential method for analysis or generative design.

\section{CONCLUSION}

Divorcing the idea of precedent and the medium of drawing from a purely representational role and reintroducing it as tool for exploration is critical for students in their education and in architecture. The utilization of Sullivan's ornament provides an ordered, rigorous and expandable method from which drawing as a tool can be advanced. This expanded utilization of drawing encourages the students to pursue drawing in their own process as a tool for discovery and evolution instead of in a purely representative manner.

As Sullivan carefully articulated in the graphic explanation of his ornament, the generative method he is pursuing is his attempt to exert control over the inorganic. By his own definition the inorganic are those things clearly altered by the will of man but still considered raw materials. To fully support these ideals one must also conclude that Sullivan's definition also expands to the geometries with which he, as well as his peers and his predecessors were working, simply circles and straight lined geometry were deemed inorganic, sinuous curvilinear geometries became associated with the organic. Curiously though throughout the generation of details he rigorously controls the inorganic but seems to relinquish control to the sinuous organicism that ultimately overtakes the rigorous geometric articulation. This holistic control over design is precisely what makes this process accessible to the students as they investigate and 'unpack' the geometric process from which these intricate ornaments are generated. This generative process engages the students with a historical reference as well as gives them insight into the utilization of architectural process, precedent and most fundamentally drawing as something other than representation.

\section{ENDNOTES}

1 Louis Sullivan, Kindergarten Chats and Other Writings, revised 1918 (New York: Wittenborn, Schult, Inc., 1947), 47.

2 Louis Sullivan, A System of Architectural Ornament According with a Philosophy of Mans Powers (New York: Press of the American Institute of Architects, Inc., 1926), Plate 3.

3 Sullivan, 1

4 The description of man's drive to create in Sullivan's eyes is inextricably linked to the biological characteristics with which we are gifted. He describes these embedded characteristics into four groups for full documentation of these characteristics see.

5 Louis H. Sullivan, A System of Architectural Ornament According with a Philosophy of Man's Powers.

6 Sullivan, Plate 3.
7 Michael Meredith et al., From Control to Design: Parametric/Algorithmic Architecture (New York: Actar-D USA, 2007), 3.

8 Simon Unwin, Analysing Architecture, 3rd edition (New York: Routledge, 2010), 18.

9 Unwin, 18.

10 Typical in gothic archway detailing a daisy wheel is a circular construction that utilizes a polar array of circles to generate internal structure reminiscent of a flower. 